\title{
Bradyrhizobia isolated from root nodules of Parasponia (Ulmaceae) do not constitute a separate coherent lineage
}

\author{
Bénédicte Lafay, ${ }^{1} \dagger$ Erika Bullier ${ }^{2}$ and Jeremy J. Burdon ${ }^{1}$ \\ ${ }^{1}$ CSIRO Plant Industry, PO Box 1600, Canberra, ACT 2601, Australia \\ ${ }^{2}$ UMR CNRS-IRD 2724, Centre IRD, 911, Avenue Agropolis - BP 64501, 34394 Montpellier \\ Cedex 5, France
}

\begin{abstract}
Rhizobial bacteria almost exclusively nodulate members of the families Fabaceae, Mimosaceae and Caesalpiniaceae, but are found on a single non-legume taxon, Parasponia (Ulmaceae). Based on their host-range, their nitrogen-fixing ability and strain competition experiments, bacterial strains isolated from Parasponia were thought to constitute a separate lineage that would account for their exceptional host affinity. This hypothesis was investigated by focusing on four isolates that are representative of the morphological and cultural types of Parasponia-nodulating bradyrhizobia. Their evolutionary relationships with other rhizobia were analysed using $16 \mathrm{~S}$ rRNA gene sequences and their nodulation properties were explored using the nodA gene as a proxy for host-range specificity. Phylogenetic analyses of the $16 \mathrm{~S}$ rRNA and nodA gene sequences revealed that bacterial isolates from Parasponia species are embedded among other bradyrhizobia. They did not cluster together in topologies based on the 16S rRNA or nodA gene sequences, but were scattered among other bradyrhizobia belonging to either the Bradyrhizobium japonicum or the Bradyrhizobium elkanii lineages. These data suggest that the ability of some bradyrhizobia to nodulate species of the genus Parasponia does not represent a historical relationship that predates the relationship between rhizobia and legumes, but is probably a more recent host switch for some rhizobia.
\end{abstract}

\section{INTRODUCTION}

Rhizobium-like bacteria nodulate almost exclusively members of the families Fabaceae, Mimosaceae and Caesalpiniaceae. The only known non-leguminous plants on which rhizobial bacteria form nodules belong to the genus Parasponia, one of 18 genera comprising the Ulmaceae (Trinick, 1973; Akkermans et al., 1978). Species of the genus Parasponia are small woody trees up to $15 \mathrm{~m}$ high with a tropical distribution that is restricted to the Malay Archipelago (Soepadmo, 1977). They are pioneer plants in mountainous areas of Indonesia, Malaysia and Papua New Guinea (Akkermans \& van Dijk, 1981) where they appear indifferent to soil fertility or type (Soepadmo, 1977; Trinick \& Hadobas, 1988). Nodulation by rhizobia-like bacteria was first reported on Parasponia rugosa Bl. in the highlands of Papua New Guinea (Trinick, 1973). Nodulation has also been described on other species within the genus Parasponia

tPresent address: UMR CNRS-IRD 2724, Centre IRD, 911, Avenue Agropolis - BP 64501, 34394 Montpellier Cedex 5, France.

The GenBank/EMBL/DDBJ accession numbers for the 16S rRNA gene and nodA gene sequences reported in this paper are AJ920034AJ920045.
(Trinick, 1976, 1980; Trinick \& Hadobas, 1988), but appears to be restricted to the genus (Akkermans et al., 1978).

The Parasponia-rhizobia association can be highly effective and levels of nitrogen fixation comparable with those observed in legume-rhizobia symbioses have been detected (Trinick, 1980). Both fast- and slow-growing rhizobia are capable of nodulating Parasponia species (Trinick \& Galbraith, 1980; Trinick \& Hadobas, 1988), but all isolates from field-collected nodules from Parasponia species correspond to slow-growing Bradyrhizobium species (Trinick, 1976; Trinick \& Hadobas, 1988). They form a group distinct from other bradyrhizobia insofar as they differ in their cultural characteristics and host-range and, notably, are generally ineffective in forming associations with legume species (Becking, 1983; Trinick \& Hadobas, 1988, 1989). Conversely, most of the legume-derived Bradyrhizobium and Rhizobium (Sinorhizobium/Ensifer) strains tested usually form poorly effective or non-effective nodules on various Parasponia species (Trinick \& Galbraith, 1980; Trinick \& Hadobas, 1988) and, even when the association is effective, nodule formation and the onset of nitrogenase activity (nitrogen fixation) are delayed (Trinick, 1988). Based on morphological and cultural criteria, Bradyrhizobium strains 
isolated from Parasponia species have been classified into five types (Trinick, 1988; Trinick \& Hadobas, 1988), but no formal taxonomic or molecular identification has been performed to ascertain the evolutionary origin of bradyrhizobia isolated from Parasponia.

Here, we address the question of the evolutionary relationships of Parasponia-derived isolates of rhizobia using the $16 \mathrm{~S}$ rRNA gene as a taxonomic index and the nodA gene as a host specificity marker.

\section{METHODS}

Rhizobial strains. The four Bradyrhizobium strains studied are those referenced in Trinick \& Hadobas (1988): NGR 231 (type 1), CP 299 (type 2), CP 315 (type 3) and CP 283 (type 5). For comparison purposes, nodA gene sequences were obtained for Australian Bradyrhizobium strains BDV5028, BDV5040, BDV5329 and BDV5111 that were nodulating Australian native legumes isolated in southeastern Australia (Lafay \& Burdon, 1998). These correspond to genospecies A, B, H and P, respectively, as designated by Lafay \& Burdon (1998).

Small-subunit rRNA gene amplification. Bacterial DNA was prepared following a previously described method (Sritharan \& Barker, 1991). Primers corresponding to positions 8-28 and 1498-1509 in the Escherichia coli 16S rRNA gene sequence (GenBank accession no. J01695) were used for amplification of the 16S rRNA genes by PCR. PCR was carried out as described by Lafay \& Burdon (1998).

nodA gene amplification. PCR products corresponding to nearly complete nodA genes were obtained using primers nodAlf/nodAlr as described by Chaintreuil et al. (2001) following the touch-down procedure used by Hannibal et al. (2000).

PCR product sequencing. PCR products were purified using the QIAquick PCR purification kit (Qiagen). Sequencing reactions were performed using the ABI PRISM BigDye Terminator v3.0 cycle sequencing ready reaction kit with AmpliTaq DNA polymerase FS (Applied BioSystems) and the products were analysed using an ABI PRISM 310 Genetic Analyzer (Applied BioSystems). Sense and antisense synthetic primers complementary to conserved eubacterial domains (Lane et al., 1985) were used to sequence both strands of the $16 \mathrm{~S}$ rRNA gene. Primers used in the amplification reaction were used in the sequencing reactions of the nodA genes.

Phylogenetic analyses. The four strains isolated from Parasponia species were compared with taxa for which both 16S rRNA and nodA gene sequences were available. These included a number of Bradyrhizobium strains as well as strains of nodulating taxa outside the Bradyrhizobium group. Among these outgroups, two strains have been shown to be able to nodulate Parasponia species, albeit ineffectively. Additionally, sequences most similar to those of the four isolates from Parasponia species [assessed by sequence similarity searches against the GenBank database using the MEGA BLAST program on the NCBI BLAST website (http://www.ncbi.nlm.nih.gov/ blast/)] and sequences for the type strains of each Bradyrhizobium species were included in the 16S rRNA gene sequence analyses. Australian Bradyrhizobium isolate WU425 and strain ANU289 (a streptomycin-resistant derivative of the Parasponia Bradyrhizobium strain CP 283) for which no 16S rRNA gene sequences were available were included in the nodA gene phylogeny reconstruction only. For both $16 \mathrm{~S}$ rRNA and nodA genes, sequences were aligned manually. In the case of $\operatorname{nod} A$, aligned translated amino acid sequences served as a guide to place gaps in the DNA sequences with respect to the codon structure of the sequences. Nearly fulllength 16S rRNA gene or nodA gene aligned sequences were used in the analyses. For the former, the extreme $5^{\prime}$ - and $3^{\prime}$-ends of the alignment were excluded, leaving 1410 nucleotides. For the latter, 594 nucleotide sites were included in the analysis with only the extreme $5^{\prime}$-ends being omitted, as their high variability prevented unambiguous alignment between taxa. Phylogenetic analyses were performed using PHYML (Guindon \& Gascuel, 2003). Maximumlikelihood phylogenies were reconstructed using the generalized time reversible model of nucleotide substitution and a discrete gamma distribution to account for variable substitution rates among sites with eight rate categories. Nucleotide frequencies, nucleotide change rate and gamma distribution shape parameters were estimated from the data. The starting tree was obtained using BIONJ. Five hundred bootstrap replications were performed to assess confidence in the topologies.

\section{RESULTS AND DISCUSSION}

We obtained sequence data for the 16S rRNA and nodA genes of four Bradyrhizobium strains isolated from Parasponia corresponding to four of the five types described by Trinick \& Hadobas (1988). Strain CC330, representative of the fifth type (type 4) defined by Trinick and Hadobas, has been lost and was not available from any culture collection.

Molecular identification based on 16S rRNA gene sequence comparisons confirmed that the four bacterial isolates capable of nodulating the non-legume genus Parasponia, $\mathrm{CP}$ 283, CP 299, CP 315 and NGR 231, belong to the genus Bradyrhizobium (Fig. 1). However, in contrast to previous assumptions (Trinick \& Hadobas, 1988, 1989), these isolates do not constitute a separate lineage that accounts for their exceptional host affinity. Indeed, our results show that the four isolates representing the morphological and cultural types of Parasponia-nodulating bradyrhizobia (Trinick,

Fig. 1. Phylogenetic characterization of bradyrhizobia isolated from the root nodules of Parasponia species (bold) based on (a) the 16S rRNA gene and (b) the common nodulation nodA gene. Taxa indicated by filled diamonds are capable of nodulating Parasponia species. Taxa highlighted by open diamonds were isolated from root nodules of Australian-native legume species. The three major divisions among bradyrhizobia, B. japonicum, $B$. elkanii and photosynthetic bradyrhizobia, are indicated by $\mathrm{J}, \mathrm{E}$ and $\mathrm{P}$, respectively. The identification of the nodA clusters follows that of Moulin et al. (2004). The bradyrhizobia 16S rRNA gene tree was rooted using Methylobacterium nodulans ORS $2060^{\top}$ (GenBank accession number AF220763), Mesorhizobium huakuii bv. loti MAFF303099 (BA000012), Rhizobium etli CFN $42^{\top}$ (U28916), Ensifer meliloti 1021 (AL591688), Ensifer sp. NGR 234 (AJ301628), Ensifer fredii USDA 257 (AY260150), Azorhizobium caulinodans ORS $571^{\top}$ (D11342), Cupriavidus taiwanensis LMG 19424 ${ }^{\top}$ (AF300324), Burkholderia caribensis TJ182 (AJ505301) and Burkholderia tuberum STM678 ${ }^{\top}$ (AJ302311) as outgroups (not shown). Percentage bootstrap supports for internal branches based on 500 replications $>50$ are shown. Bar, $0 \cdot 01$ (a) or $0 \cdot 1$ (b) substitutions per site. 

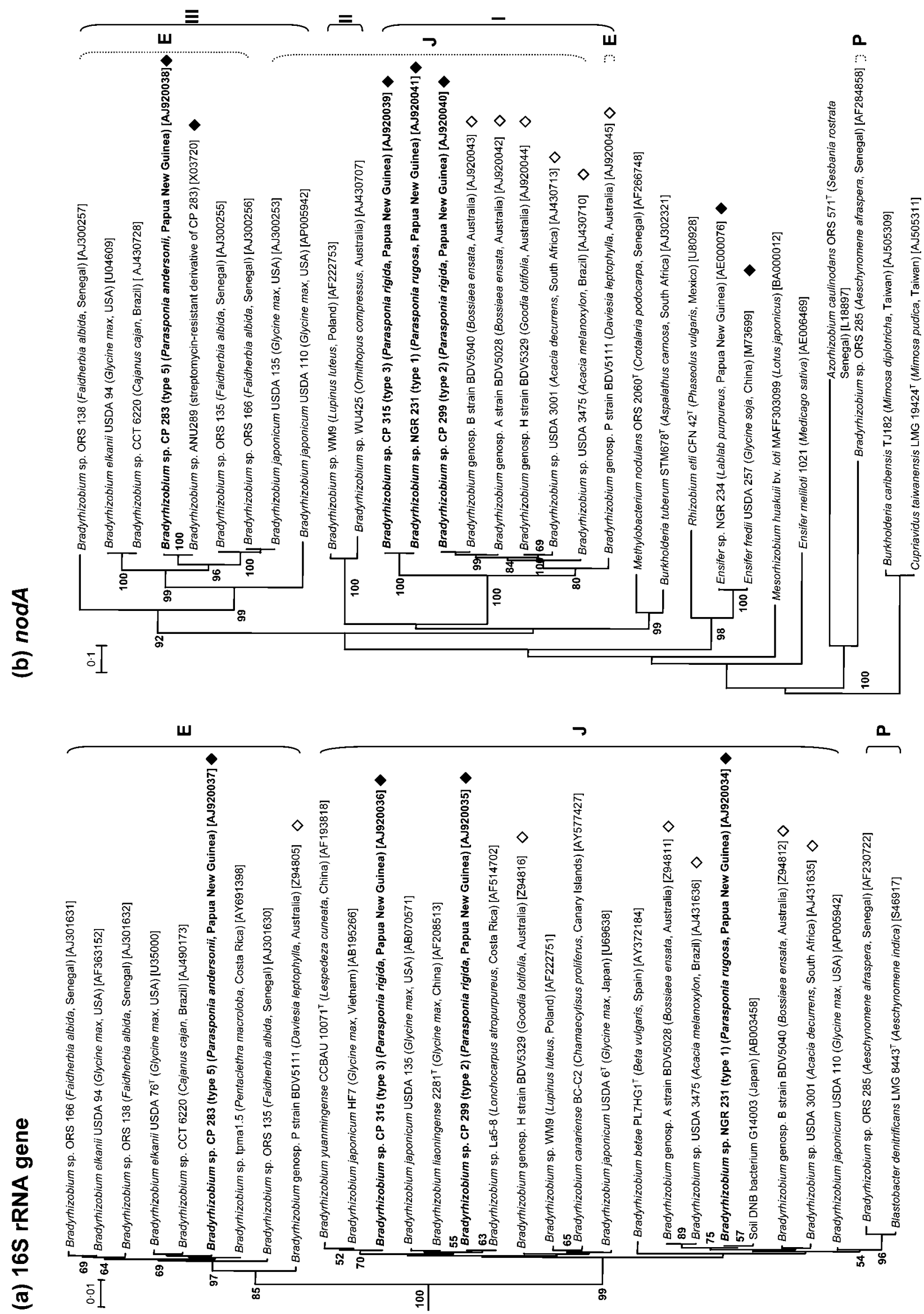
1988; Trinick \& Hadobas, 1988) can be separated into Bradyrhizobium japonicum-related and Bradyrhizobium elkanii-related genospecies. Isolates in the former group (NGR 231, CP 299 and CP 315) are as closely related to one another as they are to any species in that group, whereas the $16 \mathrm{~S}$ rRNA gene sequence of the remaining isolate (CP 283) is virtually identical to the sequence of a number of Bradyrhizobium strains (e.g. strain tpma1.5 in our phylogeny). Hence, according to this analysis of variation in the $16 \mathrm{~S}$ rRNA gene, which remains to date one of the most reliable indices for organism identification and classification (Woese, 2000), Bradyrhizobium strains derived from Parasponia are indistinguishable from those derived from a range of legume species.

What separates the former strains is that they were first isolated from nodules on the roots of the only non-legume host known to associate symbiotically with rhizobia. This raises the question as to how these bradyrhizobia acquired the ability to nodulate the exceptional host of Parasponia species. This property is not restricted to bradyrhizobia isolated from this host. A number of bradyrhizobia isolated from legumes have formed symbiotic associations with species of the genus Parasponia in glasshouse experiments (Trinick \& Hadobas, 1989). Thus, irrespective of how well they form such associations under natural conditions, they do possess the relevant genetic determinants. Such determinants also appear to exist outside the genus Bradyrhizobium. Particularly well-studied examples are found in Ensifer (formerly Sinorhizobium) sp. NGR 234, isolated from Lablab purpureus nodules in Papua New Guinea (Trinick, 1980), and Ensifer fredii USDA 257, isolated from Glycine soja in China (Keyser et al., 1982). These bacteria are characterized by exceptionally broad host-ranges (including the genus Parasponia), a state which is thought to constitute an ancestral feature in rhizobial evolution (Pueppke \& Broughton, 1999).

In rhizobia, host-range is determined by the 'nodulation genotype', i.e. the combination of nod genes present in the chromosomal or plasmid genomes (Ueda et al., 1995; Dénarié et al., 1996; Mergaert et al., 1997). Upon activation by plant flavonoids, the products of these genes interact to synthesize lipo-chitooligosaccharides, the Nod factors, which act as signals for plant tissue to differentiate and initiate nodule formation (Dénarié et al., 1996; Esseling \& Emons, 2004). Overall, host-range and nodulation gene relationships do not correlate with rhizobial phylogeny (Young \& Johnston, 1989; Dobert et al., 1994; Young \& Haukka, 1996), suggesting that horizontal transfer has played a significant role in the evolution of rhizobial symbiosis (Mergaert et al., 1997; Suominen et al., 2001). Indeed, a closer examination of nodulation gene phylogenies suggests a multi-scale evolutionary history where geographical isolation, historical descent and lateral transfer interplay with differing intensities depending on the rhizobial group under consideration (Haukka et al., 1998; Wernegreen \& Riley, 1999; Moulin et al., 2004).
Under a lateral transfer hypothesis, isolates from members of the genus Parasponia should exhibit closely related, if not identical, nodulation genes despite their divergent taxonomic positions. We investigated whether isolates from Parasponia species possessed a unique type of nodA gene. This gene belongs to the class of common nod genes which are found in all nodule-forming bacteria (Dénarié et al., 1996) and is more specifically involved in host-range determination (Ritsema et al., 1996; Roche et al., 1996). Whereas no such data had yet been obtained for any of the four types of isolates from Parasponia species, the sequence of $\operatorname{nodA}$ was available for strain ANU289, a streptomycinresistant derivative of strain CP 283 (Scott, 1986) (Fig. 1). We therefore expected CP 283 and ANU289 nodA gene sequences to be identical and indeed they almost were, grouping together within the nodA cluster III that includes the vast majority of bradyrhizobia sequences (Moulin et al., 2004). We found a single discrepancy at positions 235-236 where the strain CP 283 dinucleotide was GC instead of CG in the reported sequence for the strain ANU289 nodA gene. The discrepancy probably arose from a sequencing error, since all other available nodA gene sequences possess GC in these positions.

The nod $A$ gene sequences of the three remaining isolate types from Parasponia species were quite different from that of strain CP 283, although amongst themselves they exhibited a high level of gene sequence similarity, with strain NGR 231 and strain CP 315 nodA gene sequences differing by a single nucleotide at position 392 relative to the full-length bradyrhizobial-like nodA gene sequence (corresponding to an amino acid difference at position 127 between the translated protein sequences). They also differed from the closely related nodA genes obtained for two broad-host-range Ensifer strains that induce the formation of ineffective nodules on Parasponia species. Compared with a representative set of nodA gene sequences, they were found, nevertheless, to be bradyrhizobial in type and to form a well-supported phylogenetic group with sequences obtained from our four Australian isolates (isolated from native legumes) as well as two strains isolated elsewhere in the world from Australian-native Acacia species (Fig. 1). These latter two isolates formed a separate lineage (cluster I) in a study of bradyrhizobia nodA gene phylogeny (Moulin et al., 2004). Strains NGR 231 and CP 315 form a separate group diverging at the base of this cluster, whereas strain CP 299 branches well within that cluster, with its nodA gene being most similar to those of the six legume-derived strains constituting that group. The nodA genes of the bradyrhizobia within this cluster are characterized by an additional nucleotide triplet (TTG or GTG), which translates into an additional amino acid (Leu or Val) at position 184 in the corresponding NodA protein sequence. Interestingly, the only other available nodA gene sequence obtained from an Australian isolate (strain WU425, isolated from a nonAustralian legume species) did not possess this molecular signature and grouped within nodA gene cluster II as defined by Moulin et al. (2004). 
Whereas lateral transfer may explain the near identity of the nodA genes of strains NGR 231 and CP 315, it cannot account for all the available data regarding nodulation of Parasponia species. This suggests that non-legume nodulation probably represents a plesiomorphic character among rhizobia. Strain CP 283 provides some evidence of this. In addition to the respective phylogenetic positions of its $16 \mathrm{~S}$ rRNA and nodA genes, strain CP 283 stands apart from other isolates obtained from Parasponia species because of its ability to nodulate a wide range of tropical legumes effectively that are normally nodulated by slow-growing root-nodule bacteria. In contrast, the other isolates appear to be more specialized towards nodulation of Parasponia species (Trinick \& Hadobas, 1988). Consequently, Trinick \& Hadobas (1988) proposed that this strain nodulated Parasponia species by accident in the presence of other more competitive legume-nodulating strains. This hypothesis is further supported by data obtained for strains isolated from legume nodules. Ensifer sp. strain NGR 234 and Ensifer fredii USDA 257, both of which are capable of nodulating species of the genus Parasponia, have rhizobial-like nodA genes only remotely related to those of the novel Parasponia isolates. Most of the bradyrhizobial strains isolated from legume hosts and capable of nodulating Parasponia species studied by Trinick \& Hadobas (1989) were from Papua New Guinea, but others were from Brazil, USA and Zimbabwe. Humanmediated introduction of these strains cannot be ruled out, but it is most likely that the ability to nodulate species of the genus Parasponia is present in the genomes of isolates outside the host's geographical range.

Host-range specificity does not generally correlate with nodulation genotypes and, even though nod genes are crucial to the nodulation process, other elements participate in the specification of the host-range of rhizobia. In regard to the existence of legume-nodulating rhizobia capable of nodulating Parasponia species, our results suggest that the nodulation of Parasponia species constitutes an accident in the life history of primarily legume-nodulating rhizobial lineages. Such events probably occur because of host plant predisposition to symbiosis and nodulation. Insight into the evolution of this process requires a dual approach, combining an investigation of the dispersal and biogeography of rhizobia populations in order to discriminate between the effects of ancestry and host transfer, and an in-depth exploration of the host side of interspecies communication.

\section{ACKNOWLEDGEMENTS}

We are grateful to Dr M. J. Trinick for providing us with the Parasponia-derived Bradyrhizobium isolates characterized in this study and to Dr John Brockwell for critical reading of the manuscript.

\section{REFERENCES}

Akkermans, A. D. L. \& van Dijk, C. (1981). Non-leguminous rootnodule symbiosis with actinomycetes and Rhizobium. In Nitrogen Fixation, vol. 1, Ecology, pp. 57-103. Edited by W. J. Broughton. Oxford: Oxford University Press.
Akkermans, A. D. L., Abdulkadir, S. \& Trinick, M. J. (1978). $\mathrm{N}_{2}$-fixing root nodules in Ulmaceae: Parasponia or (and) Trema spp.? Plant Soil 49, 711-715.

Becking, J. H. (1983). The Parasponia parviflora-Rhizobium symbiosis: host specificity, growth and nitrogen fixation under various conditions. Plant Soil 75, 309-342.

Chaintreuil, C., Boivin, C., Dreyfus, B. \& Giraud, E. (2001). Characterization of the common nodulation genes of the photosynthetic Bradyrhizobium sp. ORS285 reveals the presence of a new insertion sequence upstream of nodA. FEMS Microbiol Lett 194, 83-86.

Dénarié, J., Debellé, F. \& Promé, J.-C. (1996). Rhizobium lipochitooligosaccharide nodulation factors: signaling molecules mediating recognition and morphogenesis. Annu Rev Biochem 65, 503-535.

Dobert, R. C., Breil, B. T. \& Triplett, E. W. (1994). DNA sequence of the common nodulation genes of Bradyrhizobium elkanii and their phylogenetic relationship to those of other nodulating bacteria. $\mathrm{Mol}$ Plant Microbe Interact 7, 564-572.

Esseling, J. J. \& Emons, A. M. C. (2004). Dissection of Nod factor signalling in legumes: cell biology, mutants and pharmacological approaches. J Microsc 214, 104-113.

Guindon, S. \& Gascuel, O. (2003). A simple, fast, and accurate algorithm to estimate large phylogenies by maximum likelihood. Syst Biol 52, 696-704.

Hannibal, L., Lorquin, J., D'Ortoli, N. A., Garcia, N., Chaintreuil, C., Masson-Boivin, C., Dreyfus, B. \& Giraud, E. (2000). Isolation and characterization of canthaxanthin biosynthesis genes from the photosynthetic bacterium Bradyrhizobium sp. strain ORS278. J Bacteriol 182, 3850-3853.

Haukka, K., Lindström, K. \& Young, J. P. W. (1998). Three phylogenetic groups of nodA and nifH genes in Sinorhizobium and Mesorhizobium isolates from leguminous trees growing in Africa and Latin America. Appl Environ Microbiol 64, 419-426.

Keyser, H. H., Hu, T. S., Bohlool, B. B. \& Weber, D. F. (1982). Fast-growing rhizobia isolated from root nodules of soybean. Science 215, 1631-1632.

Lafay, B. \& Burdon, J. J. (1998). Molecular diversity of rhizobia occurring on native shrubby legumes in southeastern Australia. Appl Environ Microbiol 64, 3989-3997.

Lane, D. J., Pace, B., Olsen, G. J., Stahl, D. A., Sogin, M. L. \& Pace, N. R. (1985). Rapid determination of $16 S$ ribosomal RNA sequences for phylogenetic analyses. Proc Natl Acad Sci U S A 82, 6955-6959.

Mergaert, P., Van Montagu, M. \& Holsters, M. (1997). Molecular mechanisms of Nod factor diversity. Mol Microbiol 25, 811-817.

Moulin, L., Béna, G., Boivin-Masson, C. \& Stepkowski, T. (2004). Phylogenetic analyses of symbiotic nodulation genes support vertical and lateral gene co-transfer within the Bradyrhizobium genus. Mol Phylogenet Evol 30, 720-732.

Pueppke, S. G. \& Broughton, W. J. (1999). Rhizobium sp. strain NGR 234 and $R$. fredii USDA257 share exceptionally broad, nested host ranges. Mol Plant Microbe Interact 12, 293-318.

Ritsema, T., Wijfjes, A. H. M., Lugtenberg, B. J. J. \& Spaink, H. P. (1996). Rhizobium nodulation protein NodA is a host-specific determinant of the transfer of fatty acids in Nod factor biosynthesis. Mol Gen Genet 251, 44-51.

Roche, P., Maillet, F., Plazanet, C., Debellé, F., Ferro, M., Truchet, G., Promé, J. C. \& Dénarié, J. (1996). The common nodABC genes of Rhizobium meliloti are host-range determinants. Proc Natl Acad Sci U S A 93, 15305-15310.

Scott, K. F. (1986). Conserved nodulation genes from the nonlegume symbiont Bradyrhizobium sp. (Parasponia). Nucleic Acids Res 14, 2905-2919. 
Soepadmo, E. (1977). Ulmaceae. In Flora Malesiana, ser. 1, vol. 8, pp. 31-76. Edited by C. G. G. J. van Steemis. Jakarta: Noordhoff.

Sritharan, V. \& Barker, R. H. J. (1991). A simple method for diagnosing $M$. tuberculosis infection in clinical samples using PCR. Mol Cell Probes 5, 385-395.

Suominen, L., Roos, C., Lortet, G., Paulin, L. \& Lindström, K. (2001). Identification and structure of the Rhizobium galegae common nodulation genes: evidence for horizontal gene transfer. Mol Biol Evol 18, 907-916.

Trinick, M. J. (1973). Symbiosis between Rhizobium and the nonlegume Trema aspera. Nature 244, 459-460.

Trinick, M. J. (1976). Rhizobium symbiosis with a non-legume. In First International Symposium on Nitrogen Fixation, pp. 507-517. Edited by W. E. Newton \& C. J. Nyman. Pullman, WA: Washington State University Press.

Trinick, M. J. (1980). Growth of Parasponia in agar tube culture and symbiotic effectiveness of isolates from Parasponia spp. New Phytol 85, 37-45.

Trinick, M. J. (1988). Bradyrhizobium of the non-legume, Parasponia. In Microbiology in Action, pp. 107-118. Edited by W. G. Murrell \& I. R. Kennedy. Letchworth, UK: Research Studies Press.
Trinick, M. J. \& Galbraith, J. (1980). The Rhizobium requirements of the non-legume Parasponia in relationship to the cross-inoculation group concept of legumes. New Phytol 86, 17-26.

Trinick, M. J. \& Hadobas, P. A. (1988). Biology of the ParasponiaBradyrhizobium symbiosis. Plant Soil 110, 177-185.

Trinick, M. J. \& Hadobas, P. A. (1989). Competition by Bradyrhizobium strains for nodulation of the non-legume Parasponia andersonii. Appl Environ Microbiol 55, 1242-1248.

Ueda, T., Suga, Y., Yahiro, N. \& Matsuguchi, T. (1995). Phylogeny of Sym plasmids of rhizobia by PCR-based sequencing of a nodC segment. J Bacteriol 177, 468-472.

Wernegreen, J. J. \& Riley, M. A. (1999). Comparison of the evolutionary dynamics of symbiotic and housekeeping loci: a case for the genetic coherence of rhizobial lineages. Mol Biol Evol 16, 98-113.

Woese, C. R. (2000). Interpreting the universal phylogenetic tree. Proc Natl Acad Sci U S A 97, 8392-8396.

Young, J. P. W. \& Haukka, K. E. (1996). Diversity and phylogeny of rhizobia. New Phytol 133, 87-94.

Young, J. P. W. \& Johnston, A. W. B. (1989). The evolution of specificity in the legume-rhizobium symbiosis. Trends Ecol Evol 4, 341-349. 